\title{
Optimal Point-to-Point Motion Planning of Flexible Parallel Manipulator with Multi-Interval Radau Pseudospectral Method
}

\author{
Minxiu Kong ${ }^{1}$, Zhengsheng Chen ${ }^{1}$, Chen $\mathrm{Ji}^{1}$ \\ ${ }^{1}$ Harbin Institute of Technology in Harbin, China
}

\begin{abstract}
Optimal point-to-point motion planning of flexible parallel manipulator was investigated in this paper and the 3RRR parallel manipulator is taken as the object. First, an optimal point-to-point motion planning problem was constructed with the consideration of the rigid-flexible coupling dynamic model and actuator dynamics. Then, the multi-interval Legendre-Gauss-Radau (LGR) pseudospectral method was introduced to transform the optimal control problem into Nonlinear Programming (NLP) problem. At last, the simulation and experiment were carried out on the flexible parallel manipulator. Compared with the line motion of quantic polynomial planning, the proposed method could constrain the flexible displacement amplitude and suppress the residue vibration.
\end{abstract}

\section{Introduction}

In modern industry, there are increasing demands for high speed and heavy duty industry robots, such as automobile manufacturing, food processing, petrochemistry, and logistics. However, the requirements for these robots are high repeatability, high tracking accuracy and high efficiency. Because of the characteristics of high speed and heavy duty, compliance causes by transmission systems will be much larger compared to most of other applications. Hence, the aforementioned requirements for these kind of robots will be greatly affected, thus they have to be built massive in order to strength structural stiffness from the perspective of a conventional manner, which enlarges actuator size and increases manufacturing cost. In order to balance these counterpart problems, some researchers has focused on the dynamic modelling and motion planning of flexible robots [1,2]. However, public literatures mainly focused on the serial manipulators, few similar works were found on parallel manipulators.

Studies on the optimal path planning of flexible manipulator can usually be classified into two categories, pre-defined trajectory path planning and point to point path planning. For the first kind of path planning, the trajectory is usually constrained to pass through predefined points, which were interpolated by various interpolation techniques, and one of the optimal objectives involved with the residual vibration. For the second kind of path planning, the trajectory is free of any position type constraints, for a given time, optimal objectives such as minimal effort, maximum payload, or minimum vibration was established with the satisfactory of the boundary conditions, and an optimal problem is thus established, which can be solved by direct method or indirect method. In direct methods, the original optimal control problem is converted into a parameter optimization one [3], by discretizing robot dynamic variables (states and/or controls). Then, an efficient deterministic or stochastic optimization algorithm can be applied to solve this new finite-dimensional problem. In [4] and [5], residual vibration reduction is attained approximating the joint motion profiles with splines or polynomial functions, respectively for a two-link rigidflexible manipulator and a flexible-link flexible-joint one. Famous realizations of direct method are direct shooting method [6] or direct collocation method [7]. However, to the author's knowledge, public literature on path planning of flexible manipulator with direction method hasn't considered actuator dynamics.

On the other hand, indirect methods make use of calculus of variation: necessary conditions for optimality deriving from Pontryagin's Minimum Principle (PMP) are imposed, and the resulting Two-Point Boundary Value Problem (TP-BVP) is solved, by suitable numerical techniques. Indirect methods are widely reckoned to be very accurate, particularly when a large number of elastic degrees of freedom (dofs) are present, or optimization of composite objectives is targeted [8]. In [9], Korayem has developed an algorithm for the pointto-point motion planning of a two-link flexible manipulator with revolute joints, the Euler-Lagrange formulation and AMM are used to describe the dynamics of the robot. The cases of minimum effort, minimum effort-speed, maximum payload and minimum vibration are examined, and the bvpc4 was used to solve the resulting TP-BVP. Many other works also carried out similar works with this method. One of disadvantages with bvpc4 is that it can't deals with inequality constraints. Also, this method was more sensitive to the initial values 
of variables. However, dynamic model of parallel manipulators is quite complex for the closed kinematic chains, it's impossible to do inversion operation and derivative operation of the symbol inertial matrix, even for planar parallel manipulators with more than 2 dofs, so PMP was not applicable to flexible parallel manipulators for derivative operation of state functions.

In order to constraint the amplitude of elastic displacement and suppress residue vibrations of moving platform and make full use of motors' potentials, point to point optimal path planning of a 3RRR flexible parallel manipulator was investigated. The multi-segmented LGR pseudospectral method was introduced to transform the optimal control problem to NLP. At last, simulation and experiment studies were carried out to validate the proposed method.

\section{Dynamic modelling}

The 3-RRR parallel manipulator presented in this paper is shown in Figure 1 and Figure 2. It consists of three symmetric sub-closed chains, each comprises of three consecutive revolute joint $(\mathrm{R})$. Each actuation revolute joint is driven at $O_{i}$ by a AC synchronized motor, $i=1,2,3 . \mathrm{O}_{1} \mathrm{O}_{2} \mathrm{O}_{3}$ forms a regular triangular shape with $\mathrm{R}$ as side length. The moving platform is of a regular triangular shape, and is connected to intermediate links at distal end $B_{i}$, while proximal end of the intermediate links are connected with actuation links at $A_{i}, i=1,2,3$. It's noted that the length of all links is $l_{1}$, and side length of moving platform is $h$. The coordinates of the parallel manipulator is defined in the undeformed state of the links, as shown in Figure 1,O-XY and $G-x_{G} y_{G}$ coordinate frames are attached to the base and moving platform with $O$ and $G$ as the origins respectively. Generalized coordinates for the dynamic model of the planar parallel manipulator are shown in Figure 2. Position and orientation of the moving platform with respect to the reference frame $O-X Y$ is defined as,

$$
\boldsymbol{\eta}=\left[\begin{array}{lll}
x & y & \phi
\end{array}\right]^{\mathrm{T}}
$$

Where $(x, y)$ and $\phi$ referred to position and orientation, respectively.

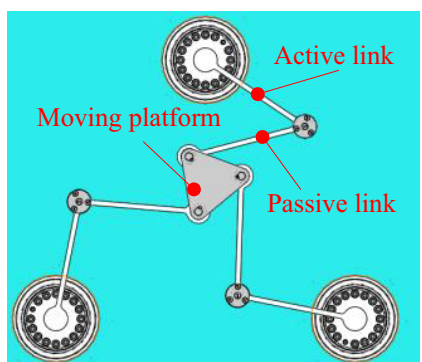

Figure 1. Structure of the 3RRR parallel manipulator

In our previous work, we found that the flexibility of the passive links can be neglected, and only flexibility of active links were accounted in this paper, an can be expressed as,

$$
\delta_{i}=\sum_{k=1}^{n} \alpha_{i}^{k} m_{i}^{k}, i=1,2,3
$$

Where $\alpha_{i}^{k}$ and $m_{i}^{k}$ is shape function and curvature of the $k$ th discretized point in the $i$ th active link. $n$ is number of discretized points of the $i$ th active link, and $n$ is chosen to be 1 in this paper. According to [10], when neglected the flexibility of passive links and added the motor and reducer model, the dynamic model of the 3RRR can be expressed as,

$$
\left[\begin{array}{ll}
\mathbf{M}_{11} & \mathbf{M}_{12} \\
\mathbf{M}_{12}^{\mathrm{T}} & \mathbf{M}_{22}
\end{array}\right]\left[\begin{array}{c}
\ddot{\boldsymbol{\eta}} \\
\ddot{\mathbf{m}}
\end{array}\right]+\left[\begin{array}{cc}
\mathbf{0} & \mathbf{0} \\
\mathbf{0} & \mathbf{K}
\end{array}\right]\left[\begin{array}{l}
\boldsymbol{\eta} \\
\mathbf{m}
\end{array}\right]+\left[\begin{array}{l}
\mathbf{f}_{1} \\
\mathbf{f}_{2}
\end{array}\right]=\left[\begin{array}{l}
\mathbf{J}_{p \theta}^{\mathrm{T}} \boldsymbol{\tau} \\
\mathbf{0}
\end{array}\right]
$$

Where $\mathbf{M}_{11}=\mathbf{M}_{11}^{\prime}+\left(I_{m}+I_{g}\right) i_{g}^{2} \mathbf{J}_{p \theta}^{\mathrm{T}} \mathbf{J}_{p \theta}$, and $\mathbf{M}_{11}^{\prime}$ is the derived inertial matrix in $\mathbf{f}_{1}=\mathbf{f}_{1}^{\prime}+\left(J_{m}+J_{g}\right) i_{g}^{2} \mathbf{J}_{p \theta}^{\mathrm{T}} \dot{\mathbf{J}}_{p \theta} \dot{\boldsymbol{\eta}}$, and $\mathbf{f}_{1}^{\prime}$ is the derived coriolis force and centrifugal force in [14]. $I_{m}$ and $I_{g}$ is rotational inertial of motor and reducers, $i_{g}$ is the reducer ratio, $\mathbf{J}_{p \theta}$ is the mapping matrix between velocity of the moving platform and angle velocity of active links. $\mathbf{K}=\operatorname{diag}\left(\left[k_{s}, k_{s}, k_{s}\right]\right)$ is the stiffness matrix, and $\boldsymbol{\tau}$ is the output torque of the reducer.

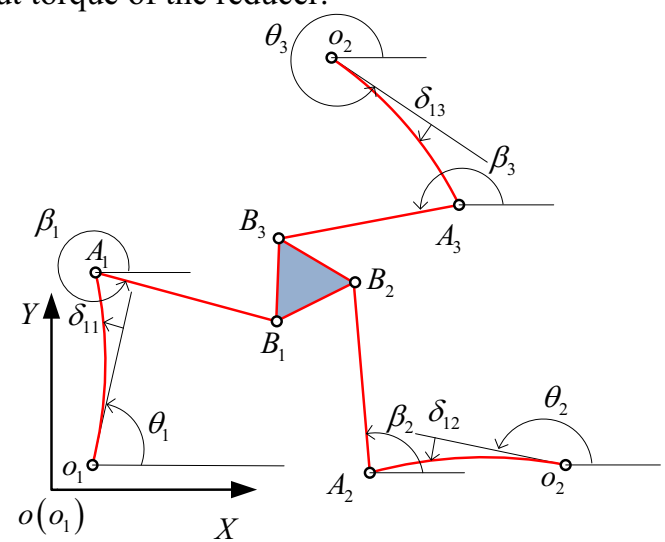

Figure 2. Coordinate frame of the $3 R R R$ parallel manipulator

\section{Point-to-point trajectory optimization}

The point-to-point motion planning problem can be described as the motion of high-speed parallel manipulator from the initial configuration $\mathbf{q}_{0}$ to the final configuration $\mathbf{q}_{f}$, with $t_{0}$ and $t_{f}$ as the starting and final time. The initial and final configuration can be expressed as,

$$
\left\{\begin{array}{l}
\mathbf{q}_{0}=\left[\begin{array}{ll}
\boldsymbol{\eta}_{0} & \mathbf{m}_{0}
\end{array}\right]^{\mathrm{T}}, \dot{\mathbf{q}}_{0}=\left[\begin{array}{ll}
\dot{\boldsymbol{\eta}}_{0} & \dot{\mathbf{m}}_{0}
\end{array}\right]^{\mathrm{T}} \\
\mathbf{q}_{f}=\left[\begin{array}{ll}
\boldsymbol{\eta}_{f} & \mathbf{m}_{f}
\end{array}\right]^{\mathrm{T}}, \dot{\mathbf{q}}_{f}=\left[\begin{array}{ll}
\dot{\boldsymbol{\eta}}_{f} & \dot{\mathbf{m}}_{f}
\end{array}\right]^{\mathrm{T}}
\end{array}\right.
$$

Where $\boldsymbol{\eta}_{0}$ and $\mathbf{m}_{0}$ is the initial state and curvatures, $\boldsymbol{\eta}_{f}$ and $\mathbf{m}_{f}$ is the final state and curvatures. While a dot means differentiation.

The optimization of motion planning lies in the utilization of the motors, the relationship between speed and torque of BECKHOFF motors was shown in Figure 3, where $\tau_{m, \text { peak }}$ and $\tau_{s}$ represents peak torque stall torque, 
$\tau_{m_{-} n}$ and $\tau_{m, \text { rated }}$ represents rated torque and nominal rated torque, while $n_{m, \text { rated }}$ and $n_{m, \text { peak }}$ represents rated speed and peak speed, respectively. The peak torque can be expressed as,

$$
\tau_{m_{-} \max }= \begin{cases}\tau_{m, \text { peak }} & \left(n \leq n_{0}\right) \\ \tau_{m, \text { peak }}-\gamma_{0}\left(n-n_{0}\right) & \left(n>n_{0}\right)\end{cases}
$$

The rapid change of torque would induce vibration to the links, to guarantee smoothness of the torque, the torque rate was introduced, and the torque was thus treated as state variables,

$$
\left\{\begin{array}{l}
\dot{\boldsymbol{\tau}}=i_{g} \mathbf{u} \\
-i_{g} \tau_{m_{-} \max } \leq \boldsymbol{\tau} \leq i_{g} \tau_{m_{-} \max } \\
-\overline{\mathbf{u}} \leq \mathbf{u} \leq \overline{\mathbf{u}}
\end{array}\right.
$$

Where $\mathbf{u}$ and $\overline{\mathbf{u}}$ represents torque rate and its maximum.

According to equation (3) and equation (6), the state variables are defined as,

$$
\left\{\begin{array}{l}
\dot{\boldsymbol{\tau}}=\mathbf{u}, \mathbf{y}_{1}=\mathbf{q}, \mathbf{y}_{2}=\dot{\mathbf{q}} \\
\mathbf{y}=\left[\begin{array}{lll}
\mathbf{y}_{\boldsymbol{\tau}} & \mathbf{y}_{1} & \mathbf{y}_{2}
\end{array}\right]^{T}
\end{array}\right.
$$

The state function can thus be expressed as,

$$
\left\{\begin{array}{l}
\dot{\mathbf{y}}_{\boldsymbol{\tau}}=i_{g} \mathbf{u} \\
\dot{\mathbf{y}}_{1}=\mathbf{y}_{2} \\
\dot{\mathbf{y}}_{2}=-\mathbf{M}^{-1} \mathbf{K} \mathbf{y}_{1}-\mathbf{M}^{-1} \mathbf{f}-\mathbf{M}^{-1} \mathbf{J} \boldsymbol{\tau}
\end{array} \Rightarrow \dot{\mathbf{y}}=\mathbf{F}_{1}(\mathbf{y}, \mathbf{u})\right.
$$

Also, the operation speed of each actuator should be within the limit of the required peak speed,

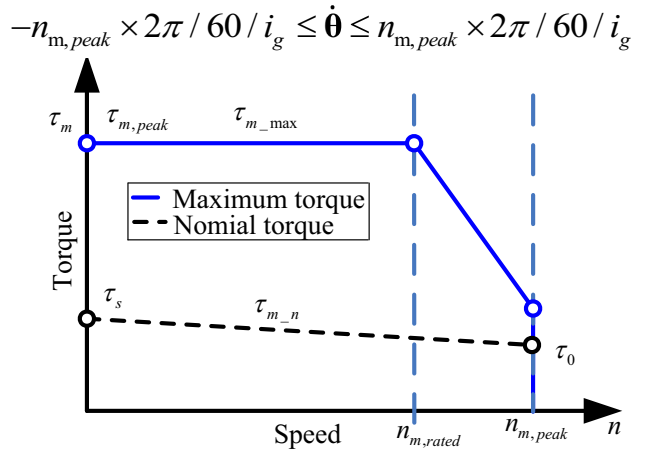

Figure 3. Speed and torque curve of Beckhoff motor

In high-speed motion, the stress of mechanical structure enduring will be quite high, so the maximum stress should be less than the allowed value,

$$
\frac{E t_{l}}{2} \max (|\mathbf{m}|) \leq[\sigma]
$$

To satisfy the motion accuracy, the elastic displacement induced by deformation and vibration of links should be constrained,

$$
|\Delta \boldsymbol{\eta}| \leq \Delta
$$

The problems may be how the moving platform moves from the initial configuration to the final configuration, which could concern with the final time $t_{f}$ fixed or free, performance of the parallel manipulator at starting or final time, energy cost during the process, and so on. An optimization objective related to the aforementioned issues should be given, and can be expressed as,

$$
J_{p}=\Phi_{1}\left(\boldsymbol{y}\left(t_{0}\right), t_{0}, \boldsymbol{y}\left(t_{f}\right), t_{f}\right)+\int_{t_{0}}^{t_{f}} \Phi_{2}(\boldsymbol{y}, \dot{\boldsymbol{y}}, \boldsymbol{u}, t) d t
$$

\section{LGR pseudospectral method}

Pseudospectral methods are a class of direct collocation method, where the optimal control problem is transcribed to a nonlinear programming problem (NLP) by parameterizing the state using global polynomials and collocating the differential-algebraic equations using nodes obtained from a Gaussian quadrature. For Legendre polynomials, the three most commonly used sets of collocation points are Legendre-Gauss (LG), Legendre-Gauss-Radau (LGR), and Legendre-GaussLobatto (LGL) points. The main differences between the three pseudospectral methods are the number of boundary points in the collocation points. In LGL, initial and final points are both included, in LGR, one of the initial and final point is included, while in LG, none of the boundary points is included. In this paper, the LGR is adopted. The Legendre polynomial with $N$ th order is expressed as,

$$
P_{N}=\frac{1}{2^{N} N !} \frac{d^{N}}{d x^{N}}\left(\tau^{2}-1\right)^{N} \quad(-1 \leq \tau \leq 1)
$$

The Gauss integral points in LGR are the roots of $P_{N}+P_{N-1}=0$. From the above equation, the number of roots in Legendre polynomial with $N$ th order is $N$. The initial boundary $\tau_{1}=-1$ is included in the roots, but in the motion planning of robotics with flexible links, the final boundary points is usually given, which will be included with the $N$ LGR points in the approximation of the states.

The period of the point-to-point motion was divided into $K_{1}$ intervals, the time points are $t_{0}<t_{1}<t_{2}<$ $\ldots<t_{K_{1}}=t_{f}$. For arbitrary interval $k$ in $K_{1}$, the time $t \in\left[t_{k-1}, t_{k}\right]$ can be expressed as,

$$
t=\frac{t_{k}+t_{k-1}}{2}+\frac{t_{k}-t_{k-1}}{2} \tau, \tau \in[-1,1]
$$

The approximation of state variables can be written as,

$$
\mathbf{y}^{(k)}(\tau)=\sum_{i=1}^{N_{k}+1} L_{i}^{(k)}(\tau) \mathbf{Y}^{(k)}\left(\tau_{i}\right)=\sum_{i=1}^{N_{k}+1} L_{i}^{(k)} \mathbf{Y}_{i}^{(k)}
$$

Where $N_{k}$ is number of Gauss integral points in LGR, $\mathbf{Y}_{i}^{(k)}$ represents state variables in interval $k$ at the integral point $\tau_{i} . L_{i}^{(k)}$ is Lagrange interpolation polynomial, and can be expressed as,

$$
L_{i}^{(k)}=\prod_{j=1, i \neq j}^{N_{k}+1} \frac{\tau-\tau_{j}}{\tau_{i}-\tau_{j}}
$$

Differentiate (15) with respect to time, derivatives of state variables can be written as,

$$
\dot{\mathbf{y}}^{(k)}(\tau)=\sum_{i=1}^{N_{k}+1} \dot{L}_{i}^{(k)}(\tau) \mathbf{Y}^{(k)}\left(\tau_{i}\right)=\sum_{i=1}^{N_{k}+1} \dot{L}_{i}^{(k)} \mathbf{Y}_{i}^{(k)}
$$

In LGR pseudospectral method, the constraints of state equation should be satisfied in $N_{k}$ integral points, 


$$
\begin{aligned}
& \sum_{i=1}^{N_{k}+1} D_{j i}^{(k)} \mathbf{Y}_{i}^{(k)}-\frac{t_{k}-t_{k-1}}{2} \mathbf{F}_{1}\left(\mathbf{Y}_{i}^{(k)}, \mathbf{U}_{i}^{(k)}, \tau_{i}^{(k)} ; t_{k-1}, t_{k}\right)=0 \\
& k=1,2 \cdots K, j=1,2 \cdots N_{k}
\end{aligned}
$$

Where $\mathbf{U}_{i}^{(k)}$ represents torque rate in interval $k$ at the integral point $\tau_{i}, D_{j i}^{(k)}=\dot{L}_{i}^{(k)}\left(\tau_{j}\right)$ is $N_{k} \times\left(N_{k}+1\right)$ LGR differentiation matrix, which is non-square matrix because of the addition of final boundary point for the approximation of states. According to [10], the first $N_{k}$ column of differentiation matrix can be written as,

$$
D_{j i}^{(k)}= \begin{cases}\frac{-\left(N_{k}-1\right)\left(N_{k}+1\right)}{4} & i=j=1 \\ \frac{P_{N_{k}-1}\left(\tau_{j}\right)}{P_{N_{k}-1}\left(\tau_{i}\right)} \frac{1-\tau_{i}}{1-\tau_{j}} \frac{1}{\tau_{j}-\tau_{i}} & i \neq j, \quad 1 \leq i, j \leq N_{k} \\ \frac{1}{2\left(1-\tau_{j}\right)} & 1 \leq i=j \leq N_{k}\end{cases}
$$

Where $P_{N_{k}-1}$ is the $N_{k}-1$ order Legendre polynomial.

When the constant 1 is approximated by LGR, the derivative of the approximation must be zero, that is $\mathbf{D}_{N_{k}+1}^{(k)}+\mathbf{D}_{1: N_{k}}^{(k)} \mathbf{1}=\mathbf{0}$, where $\mathbf{D}_{1: N_{k}}^{(k)}$ is a square matrix comprised of the first $N_{k}$ column of differentiation matrix, while $\mathbf{D}_{N_{k}+1}^{(k)}$ is the $N_{k}+1$ column. Because the differentiation matrix has no relation with the approximated variables, so $\mathbf{D}_{N_{k}+1}^{(k)}$ can be expressed as,

$$
\mathbf{D}_{N_{k}+1}^{(k)}=-\mathbf{D}_{1: N_{k}}^{(k)} \mathbf{1}
$$

The constraints of operation speed expressed by (9) in LGR integral points can written as,

$-n_{\mathrm{m}, \text { peak }} \times 2 \pi / 60 / i_{g} \leq \mathbf{J}_{p \theta} \sum_{i=1}^{N_{k}+1} D_{j i} \mathbf{Y}_{1 i}^{(k)} \leq n_{\mathrm{m}, \text { peak }} \times 2 \pi / 60 / i_{g}$

Where $\mathbf{Y}_{1 i}^{(k)}$ represents position and posture of the moving platform in interval $k$ at the integral point $\tau_{i}$.

The equation (10) and (11) at LGR points can be rewritten as,

$$
\left\{\begin{array}{l}
\left|\mathbf{J}_{p \theta}^{-1} \boldsymbol{\alpha}_{1} \mathbf{Y}_{2 i}^{(k)}\right| \leq \Delta \\
\frac{E t_{l}}{2}\left|\mathbf{Y}_{2 i}^{(k)}\right| \leq[\sigma]
\end{array}\right.
$$

Where $\boldsymbol{Y}_{2 i}^{(k)}$ represents position and posture of the moving platform in interval $k$ at the integral point $\tau_{i}$.

According to (6), the torque and torque rate can be written as,

$$
\left\{\begin{array}{l}
-i_{g} \tau_{m_{-} \max } \leq \mathbf{Y}_{\boldsymbol{\tau} i}^{(k)} \leq i_{g} \tau_{m_{-} \max } \\
-\overline{\mathbf{u}} \leq \mathbf{U}_{i}^{(k)} \leq \overline{\mathbf{u}}
\end{array}\right.
$$

The cost function of (12) can be written as,

$$
\begin{aligned}
J_{p}= & \Phi_{1}\left(\mathbf{Y}_{1}^{(1)}, t_{0}, \mathbf{Y}_{N_{k}+1}^{\left(K_{1}\right)}, t_{K_{1}}\right)+ \\
& \sum_{k=1}^{K_{1}} \frac{t_{k}-t_{k-1}}{2} \sum_{i=1}^{N_{k}} a_{\mathrm{LRG}, i} \Phi_{2}\left(\mathbf{Y}_{i}^{(k)}, \mathbf{U}_{i}^{(k)}, \tau_{i}^{(k)} ; t_{k-1}, t_{k}\right)
\end{aligned}
$$

Where $a_{\mathrm{LRG}, i}$ is weight coefficient of LGR Gauss integral points, and can be written as,

$$
a_{\mathrm{LRG}, i}= \begin{cases}\frac{2}{\left(N_{k}+1\right)^{2}} & i=1 \\ \frac{1}{\left(N_{k}+1\right)^{2}} \frac{1-\tau_{i}}{\left(P_{N_{k}-1}\left(\tau_{i}\right)\right)^{2}} & i \neq 1\end{cases}
$$

With the multi-interval pseudospectral method, the optimal control problem has been transformed into nonlinear programming problem, and can be solved by the mature software SNOPT.

\section{The simulation}

A simulation study of point-to point motion planning of the 3RRR flexible parallel manipulator will be carried out, and the optimal control problem will be solved by the aforementioned multi-interval LGR pseudospectral method. The parameters used in the simulation were the same as our previous paper [10], and the corrected and new parameters are shown in Table 1.

Most of existing work about the motion planning of manipulators with flexible links chose strains, driving torques or motion error as the cost function in given time, but they didn't show how to choose the time. To solve this problem, the motion time was chosen as the cost function, as shown in (26). The elastic displacement of the moving platform in $X$ direction and $Y$ direction is set below $1.5 \mathrm{~mm}$ and $0.2 \mathrm{~mm}$.

$$
J_{p}=\int_{0}^{t_{f}} 1 d t
$$

Table 1 Specification for the 3RRR parallel manipulator

\begin{tabular}{|c|c|c|}
\hline Parameters & Value & Explanation \\
\hline$t h \times h e$ & $5 \mathrm{~mm} \times 30 \mathrm{~mm}$ & Link thickness $\times$ Link thickness \\
\hline$i_{g}$ & 20 & Reducer ratio \\
\hline$I_{m}+I_{g}$ & $284.1 \mathrm{~kg} \mathrm{~mm}^{2}$ & Motor and reducer's inertial \\
\hline
\end{tabular}

In the simulation, approximation accuracy is set as $\varepsilon_{d 2}=1 \times 10^{-6}$, the period is divided into 6 intervals, and the number $N_{k}$ for each interval is 10 , iterations for the optimization is 2000 . The initial configuration is set as $\mathbf{q}_{0}=\left[\begin{array}{lll}187.5 & 187.5 / \sqrt{3} & \mathbf{0}_{1 \times 4}\end{array}\right]$ and $\dot{\mathbf{q}}_{0}=\left[\begin{array}{ll}\mathbf{0}_{1 \times 6}\end{array}\right]$, and the final configuration is set as $\mathbf{q}_{f}=\left[\begin{array}{lll}217.5 & 187.5 / \sqrt{3} & \mathbf{0}_{1 \times 4}\end{array}\right]^{\mathrm{T}}$ and $\dot{\mathbf{q}}_{f}=\left[\begin{array}{ll}\mathbf{0}_{1 \times 6}\end{array}\right]$. The allowed stress of links is set as $[\sigma]=355 \mathrm{MPa}$, The limit torque of the output end of reducer is set as $-150 \mathrm{Nm} \leq \mathbf{Y}_{\boldsymbol{\tau} i}^{(k)} \leq 150 \mathrm{Nm}$, the torque rate in (5) is $\gamma_{0}=0.12 \mathrm{Nm} / \mathrm{rpm}$, torque rate for optimization is set as $-0.001 \mathrm{Nm} / \mathrm{s} \leq \dot{\mathbf{Y}}_{\boldsymbol{i}}^{(k)} \leq 0.001 \mathrm{Nm} / \mathrm{s}$, the range for the speed of the motor is $-6000 \mathrm{rpm} \leq n_{\text {m,peak }} \leq 6000 \mathrm{rpm}$.

The result is shown as Figure 4, the optimized time $t_{f}$ is $\left.0.0508 \mathrm{~s}, \mathrm{a}\right)$ and $\mathrm{b}$ ) is the position and velocity of the moving platform in $X$ direction, the position is like the $\mathrm{S}$ 
curve except for local fluctuation, and the velocity curve shows that the initial and final point of velocity and its derivatives are zeroes, which guarantee smoothness of the trajectory. The elastic displacement of the moving platform in e) was within the constraint. Also, torques at LGR integral points are satisfied in constraint.

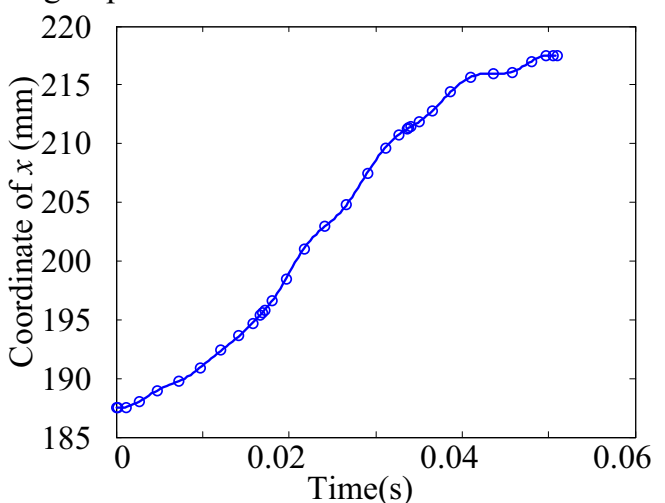

a) Coordinate of moving platform in $X$ direction

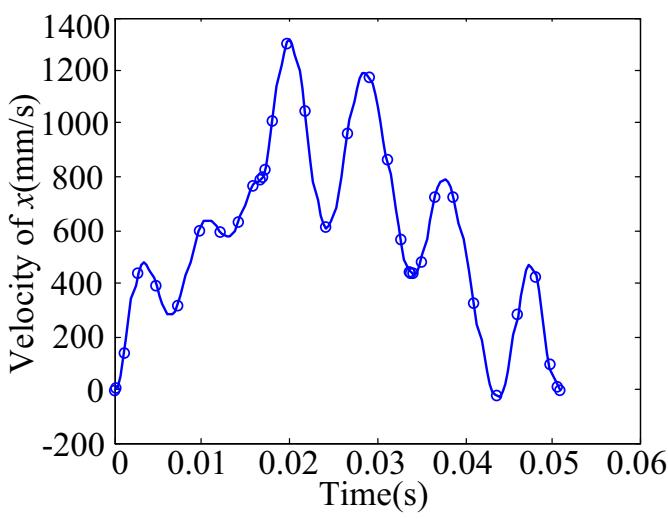

b) Velocity of moving platform in $X$ direction

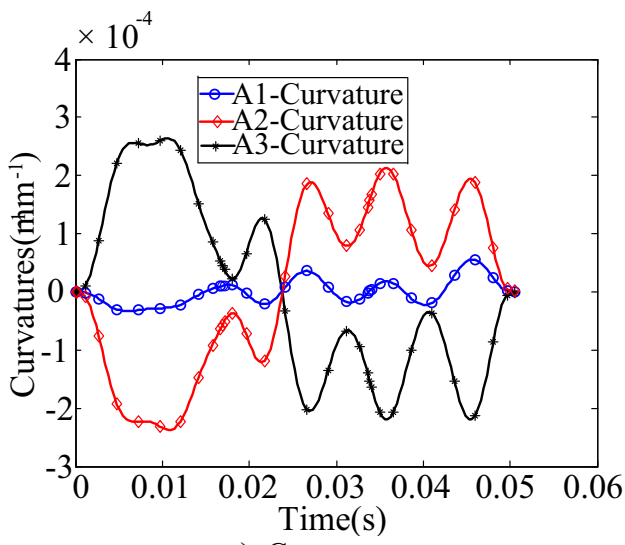

c) Curvatures

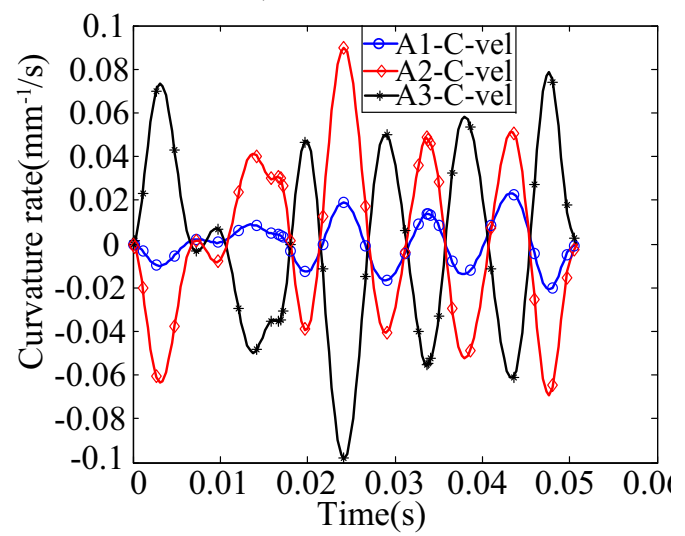

d) Curvature velocities

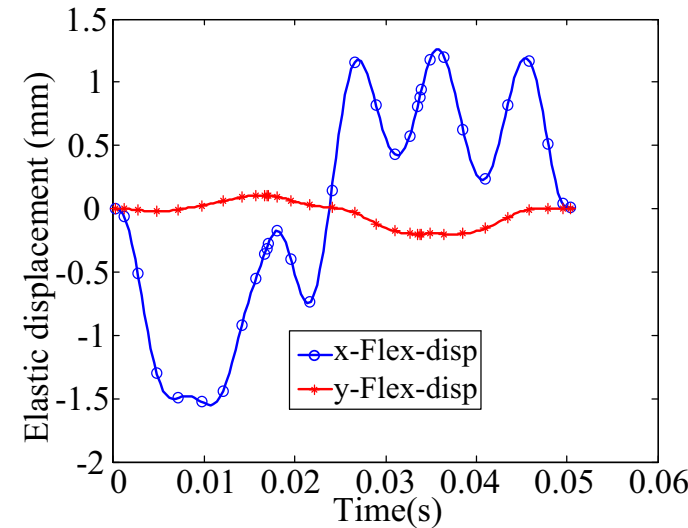

e) Flexible displacement of the moving platform

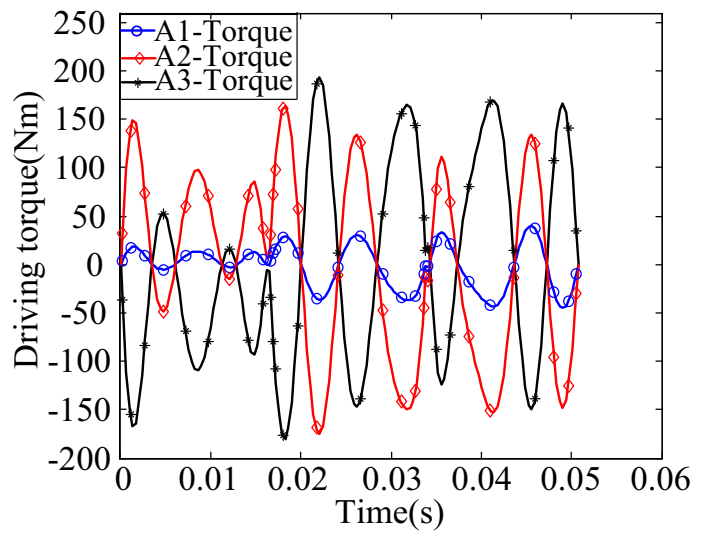

f) Driving torques

Figure 4. The optimization result of pseudospectral method

\section{Experiments}

To validate the proposed method, the prototype of the 3RRR parallel manipulator was established. The industrial computer, real-time operating system and highspeed communication bus control structure was adopted, the optimized trajectory can then be programmed, and the strain of each link can then be tested by the strain amplifier, which can transmit the information into the controller through bus coupler. To see the effect of the optimized trajectory, the quantic polynomial with the same operation time was introduced to make a comparison study, the equation is,

$$
p_{x}=187.5+30\left(6 t^{5} / t_{d}^{5}-15 t^{4} / t_{d}^{4}+10 t^{3} / t_{d}^{3}\right)
$$

Where $t_{d}$ is $0.0508 \mathrm{~s}$.

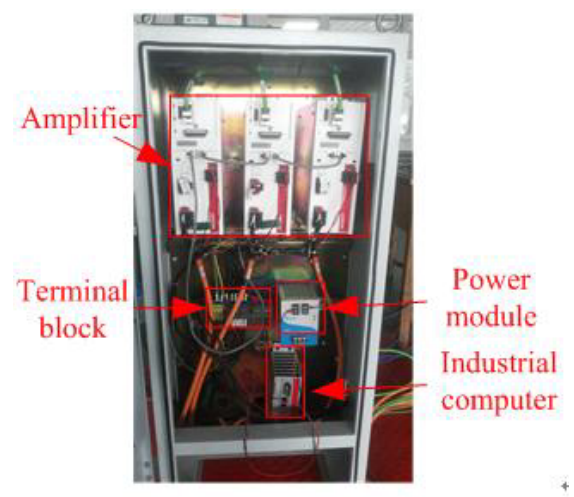

a) The control hardware for the manipulator 


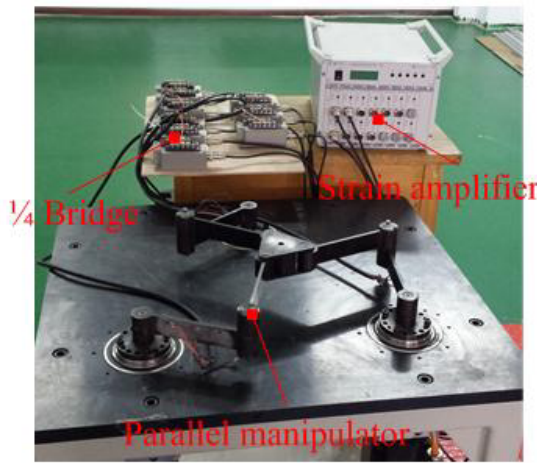

b) The manipulator and the strain amplifier

Figure 5. Test setup for the experiment

The strains of all links with the optimized trajectory and quantic polynomial planning were shown in Figure 6. It shows that the maximum strains reduced from more than $5 \times 10^{-4}$ with quantic polynomial planning to less than $4 \times 10^{-4}$ with pseudospectral method, also the residue vibration with quantic polynomial planning is more than $1.5 \times 10^{-4}$, while the value is less than $2 \times 10^{-5}$ in pseudospectral method. We can see that the aim for constraining the amplitude of elastic displacement amplitude and suppressing the residue vibration is realized.

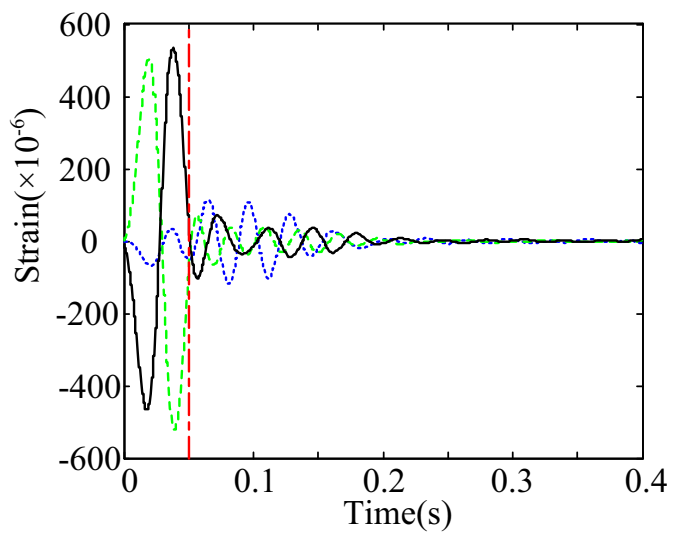

a) Strains of all links with quantic polynomial planning

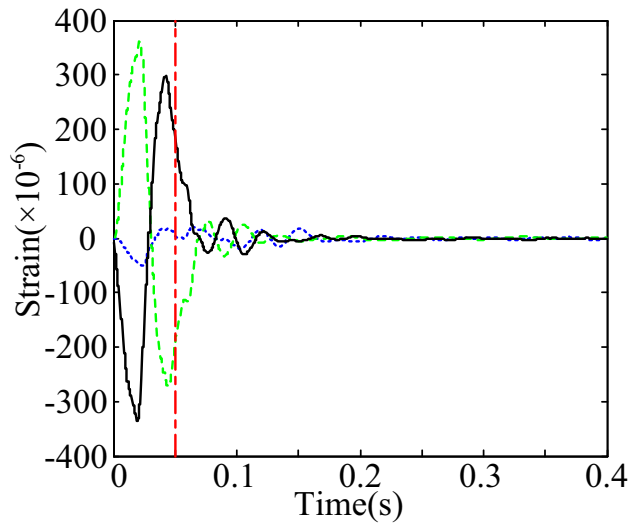

b) Strains of all links with pseudospectral method

Figure 6. Strains of all links with different planning methods

\section{Conclusions}

In this paper, optimal point-to-point motion planning of a 3RRR parallel manipulator has been investigated. Based on our previous work on the dynamic modeling of the 3RRR parallel manipulator, an optimal point-to-point motion planning problem was constructed with consideration of the flexible dynamic model and actuator dynamics, and the multi-interval (LGR) pseudospectral method was introduced to transform the optimal control problem into NLP. At last, the simulation and experiment were carried out on the flexible parallel manipulator, compared with the line motion of quintic polynomial, the proposed method show significant advantages on constraint the flexible displacement amplitude and suppress the residue vibration.

\section{References}

1. Abe, A., Mechanism and Machine Theory, 44, 9 (2009).

2. Korayem, M.H., Rahimi, H., and Nikoobin, A., Applied Mathematical Modeling, 36, 7(2012).

3. Hull, D.G., Journal of Guidance, Control, and Dynamics, 20, 1(1997).

4. H Kojima, T Kibe, Proceedings of 2001 IEEE/RSJ International Conference on Intelligent Robots and Systems, 2001.

5. Park, K.-J., Journal of Sound and Vibration, 275, 3 (2004)

6. von Stryk, D.M.O., Numerical solution of optimal control problems by direct collocation, 1993.

7. Hargraves, C., and Paris, S., 4, 1986.

8. Kirk, D.E., Optimal control theory: an introduction, 2012.

9. Korayem, M.H., Nikoobin, A., and Azimirad, V., Robotica, 27, 6 (2009).

10. Z. Chen, M. Kong, C. Ji, et al, PROCEEDINGS OF THE INSTITUTION OF MECHANICAL ENGINEERS PART C-JOURNAL OF MECHANICAL ENGINEERING SCIENCE, 229, 4 (2015). 